F. Blumenthal, Bericht üb. d.'Tätigkeit im Universitätsinstitut f. Krebsforsch. 13

\title{
3. Die Abteilung für physikalische Behandlungsmethoden.
}

\author{
Von Dr. med. J. Tugendreich.
}

Die Gesamtzahl der seit Einrichtung des Ambulatoriums und der Abteilung für physikalische Behandlungsmethoden im Krebsinstitut vom 16. Juni 1915 bis 15. Juni 1916 aufgenommenen Patienten betrug 580. Von diesen wurden über die Hälfte, da wir nichts bei ihnen feststellen konntenl, was für die Diagnose einer Geschwulst sprach, den entsprechenden anderen Kliniken und Polikliniken überwiesen. Von den übrigen wurden 230 auf unserer Abteilung in ambulante Behandlung genommen. Bei den nicht von uns behandelten Krebskranken und -verdächtigen handelte es sich um zum Teil noch operable Carcinome, die auf die chirurgische Klinik verlegt wurden, zum Teil waren es Frauen mit Tumoren der Geschlechtsorgane, die wir der Frauenklinik überwiesen haben.

Unter den 230 mit Röntgenstrahlen behandelten Patienten hatten bösartige Geschwülste an folgenden Organen:

98 Mamma,

30 Milz, Drüsen und Mediastinum,

19 Magen, Darm,

15 Kopf, Gesicht, Lippen,

14 Knochen und Muskel,

12 Rektum,

9 Zunge,

Von diesen waren 60 Männer und 170 Frauen. Fast die Hälfte aller mit Röntgenstrahlen behandelten Patienten waren demnach Frauen mit Nlammacarcinomen. Sie verteilen sich in folgender Weise:

70 Fälle ron postoperativen Rezidiven und Metastasen,

$19 "$ von inoperablen Carcinomen,

9 , von noch operablen Carcinomen

98 Fälle, dazu kommen noch

insgesamt 105 Fälle.

von postoperativen prophylaktischen Bestrahlungen

In allen iibrigen Fällen hatten wir es fast ausschließlich mit inoperablen Tumoren, wobei außer den primären Geschwülsten mehr oder weniger ausgedehnte Metastasen klinisch oder röntgenologisch nachzuweisen waren; oder mit postoperativen Rezidiven zu tun.

Mit Ausnahme der wenigen Patienten mit anscheinend noch operablen Mammacarcinomen, die trotz unserer dringlichen Ermahnungen sich keiner Operation unterziehen lassen wollten, befanden sich alle unsere iubrigen Patienten in einem sehr stark fortgeschrittenen Stadium der Krankheit. 
14 F. Blumenthal, Bericht äb.d. 'lätigkeit im Universitätsinstitut f. Krebsforsch.

Unsere Aufgabe mußte sein - wollten wir mit Röntgenstrahlen einen therapeutischen Erfolg erzielen - eine möglichst intensive und harte Strahlung in Anwendung zu bringen.

Im Ralımen dieser Mitteilung, die lediglich einen Bericht über unsere erste einjährige Tätigkeit darstellen soll, können wir auf einige wichtige und ihrer Lösung harrende Probleme der Strahlentherapie nicht eingehen, so die praktisch bedeutsame Frage, ob der ganze Strahlenkomplex, die kurz- und langwelligen Strahlen in gleicher Weise pathologisches radiolabiles Gewebe angreift, und die verschiedenen Strahlen sich nur durch ihre geringere oder stärkere Durchdringungsfähigkeit voneinander unterscheiden, - oder sind tatsächlich mittelweiche und harte Strahlenkomplexe verschiedene röntgenotherapeutische Agentien mit verschiedenen biologischen Wirkungen? Ist letzteres der Fall - und wir haben uns im Krebsinstitut nach diesem Prinzip gerichtet -, so kann man nicht die Oberflächentherapie der Tiefentherapie entgegenstellen, sondern man muß ron einer "Therapie mit mittellangwelligen Strahlen" und solcher "mit kurzwelligen Strahlen" sprechen. - Gelingt es der Siemens \& Halske A.-G., durch ihren angekündigten neuen Apparat für hochgespannten Gleichstrom und die Glühkathodenröhre die Heterogenität der unfiltrierten Strahlung merklich herabzusetzen und dabei auch sehr harte Strahlen zu erzeugen, so wird es wahrscheinlich möglich sein, die Frage der biologischen Wirkungsspezifität verschiedener Strahlen experimentell zu erforschen.

Vorläufig standen uns für unsere Zwecke zwei Röntgenapparate zur Verfïgung:

ein Apex-Apparat der Reiniger, Gebbert \& Schall A.-G. und

ein Triplex-Apparat der Firma Siemens \& Halske.

Beide Apparate im Verein mit geeigneten Röhren, die wir noch später kurz besprechen werden, erfüllen im großen und ganzen die Bedingungen, die man gegenwärtig an die Leistungen der Röntgenapparate stellen kann.

Diese Forderungen sind:

1. Möglichkeit eines Dauerbetriebs bei einer Röhrenbelastung von 2-3 Milliampère.

2. Hohe Spannung, um einen möglichst großen Anteil an harten Strahlen in der Röhre zu erzeugen.

3. Vollkommen schließungslichtfreier Betrieb.

Beide Apparate besitzen neben dem Induktor einen QuecksilberGasunterbrecher.

Beim Apex-Apparat sorgt ein elektrischer Ventilator für eine dauernde Kühlung des Induktors und der Luft im Schrankinnern, und ein synchron mit dem Unterbrecher laufender Nadelschalter erfüllt die Aufgabe, verkehrt gerichtete Ströme, i. e. Schließungsströme, von der Röhre fernzuhalten. 
Ein ebenso absolutes schließungsfreies Arbeiten und eine hohe Spannung wird im Triplex-Apparat durch eine rotierende Schaltvorrichtung, die an die bekannten Hochspannungsgleichrichter erinnert, gewährleistet. Die rotierende Schaltvorrichtung, die mechanisch mit dem Unterbrecher, elektrisch mit der Sekundärspule des Induktors in Verbindung steht, besteht aus 3 Kontaktarmen, welche an feststehenden, in 4 Stockwerlsen übereinander angebrachten Metallsegmenten vorbeilaufen und sie miteinander verbinden. Der Primärstrom wird durch den Unterbrecher geschlossen, wenn die 3 Kontaktarme keine Verbindung zwischen den Segmenten herstellen, und da die Röhre an den einen Pol des Induktors über die Segmente angeschlossen wird, so kann kein Strom durch die Röhre fließen. Nur in dem Augenblick, wenn der Primärstrom durch den Unterbrecher geöffnet wird, gleiten die Kontaktarme zwischen den Segmenten durch und die Röhre erhält Strom. In dieser Weise können Stromimpulse nur in einer Richtung durch die Röhre treten. Dabei sind die Segmente so lang bemessen, daß nur eine sehr kurze Stromschlußdauer eintritt, und es kommt dadurch nur das Spannungsmaximum in Betracht. Es wird hierdurch erreicht, daß der Strahlenkomplex in der Röhre reich an harten Strahlen ist.

Der Apparat ist ferner so eingerichtet, daß mit ihm eine, zwei und drei Röhren mit je einem, zwei oder drei Stromimpulsen betrieben werden kann. Wir arbeiten therapeutisch stets mit einer Röhre und drei Stromimpulsen. Es gelingt uns, in dieser Weise auch für diagnostische Zwecke bei Verwendung einer Verstärkungsfolie kurzzeitige Thorax- und Magenaufnahmen zu machen.

Für therapeutische Bestrahlungen gebrauchen wir ausschließlich das Siederohr der Firma C. H. F. Müller in Hamburg mit Osmofernregenerierung. Die für die konstante Erzeugung eines harten Strahlenkomplexes so wichtige Vorrichtung zur Kühlung der Antikathode ist bei dieser Röhre in glänzendster Weise gelöst. Bezüglich der Konstruktion dieser Röhre und ihrer Vorzüge verweisen wir auf die sehr. interessante Mitteilung von Bucky in Nr. 34 der Münch. med. Wochenschr. 1915. Wir wollen hier nur erwähnen, daß die Röhren, nachdem sie sich eine kurze Zeit eingearbeitet haben, bei unserem Dauerbetrieb von etwa 8 Stunden täglich (am "Triplex" gemessen) eine konstante Härte von etwa $1,7 \mathrm{~cm}$ Halbwertschicht nach Christen aufweisen, und daß die Lebensdauer unter diesen Verhältnissen bei rationeller Behandlung durchschnittlich 110 Bestrahlungsstunden beträgt. Eine Röhre, die noch bei uns in Gebrauch ist, hat 160 Bestrahlungsstunden hinter sich.

Als Härter benutzen wir $5 \mathrm{~mm}$ Aluminium. Wir erhalten dann einen Härtegrad von etwa $3 \mathrm{~cm}$ Halbwertschicht in Wasser. Der Härtegrad ist gerade noch an dem sich im Handel befindenden Härtemesser von Christen ablesbar. 
Bei einer sekundären Stromstärke von 2 Milliampère und einem Antikathoden-Hautabstand von $24-26 \mathrm{~cm}$ erhalten wir in 15 Minuten eine $\mathrm{Ober}$ flächendosis, die hinter $5 \mathrm{~mm}$ Aluminium nach Sabouraud gemessen etwa $1 / 3$ bis $1 / 2$ Volldosis beträgt, mit anderen Worten einen Strahlenkomplex, der verhältnismäßig wenig mittelweiche Strahlen enthält.

Mangels eines auch nur einigermaßen zuverlässigen Dosimeters für die so gewonnene ganz harte Strahlung und bei der leicht konstant zu haltenden Härte unserer Röhren begnügen wir uns zur Bezeichnung der Quantität der applizierten Strahlen mit dem Produkte aus Intensität und Zeit und rechnen nach Milliampère-Minuten. Nach unseren Erfahrungen können wir bei unserem Krankenmaterial nur solche Patienten in einer Statistik verwerten, die bei der bei uns üblichen konzentrischen Vielfelderbestrahlung, Felder von je 5 bis $8 \mathrm{~cm}$ Durchmesser, mindestens 300 Milliampère-Minuten abbekommen haben.

Die größte verabreichte Strahlenmenge bei einem Patienten betrug bis jetzt 2150 Milliampère-Minuten.

Abgesehen davon, daß die Patienten, die zu uns kommen, sich mit wenigen Ausnahmen in einem von vornherein therapeutisch aussichtslosen Zustand befinden, gehören die Tumoren, die wir zu bestrahlen hatten, zum größten Teil bekanntlich nicht zu den radiolabilsten.

Die Rezeptivität normaler Zellen der Strahlung gegenüber ist ja im allgemeinen eine viel geringere als die pathologischer Zellen und insbesondere der Geschwulstzellen. Daß die Röntgenstrahlen und ganz besonders die kurzwelligen harten Strahlen kein "Kaustikum" sind, die wahllos alle Gewebe, die sie erreichen, zerstören, ist hinreichend experimentell und praktisch erwiesen. Aber auch innerhalb der verschiedenen Geschwiulste besteht eine Verschiedenheit der Sensibilität der lebenden Geschwulstelemente den therapeutischen Bestrahlungen gegenüber. Wenn auch bis jetzt nicht alle Lrsachen für die geringere oder stärkere Radiolabilität der Geschwulstzellen genügend erforscht sind (in Betracht kommen u. a. Alter, Lokalisation, Gruppierung, Art usw. der Zellen), so wissen wir erfahrungsgemäß, daß z. B. die aus lymphatischen Zellen bestehenden Tumoren zu den radiolabilen gehören (Milztumoren). Ebenso verhalten sich die ektodermalen Zellen der Regio vulvo-vaginalis (Portiocarcinom). Andererseits verhalten sich die Carcinome der Zunge. des Magens und Darms sehr häufig refraktär und auch die Radiolabilität der Mammatumoren ist im allgemeinen eine verhältnismäßig geringe. Demgemäß, können eklatante Heilresultate bei einem Krankenmaterial wie dem unserigen und bei dem jetzigen Stande der Strahlentherapie nicht erwartet werden. Von den 223 bestrahlten Patienten müssen wir nach dem obigen Begriff der Minimalmenge an Strahlen als ungenügend Bestrahlte 97 abziehen, und 38 Fälle können wir als vorläufig gebessert bezeichnen. 
F. B lumenthal, Bericht üb. d. Tätigkeit im Universitätsinstitut f. Krebsforsch. 17

Unter vorläufiger Besserung verstehen wir ein vollständiges Zurïkgehen der fühlbaren und sichtbaren Tumoren bei jetzt noch andauerndem subjektiven Wohlbefinden der Patienten. Diese 38 Fälle rekrutieren sich aus:

20 Mammacarcinomen, und zwar:

14 postoperative primäre Rezidive und Metastasen,

3 inoperable Carcinome,

2 operable Carcinome,

1 Magentumor;

2 Rektumcarcinomen,

2 Zungencarcinomen,

3 Kopf-, Gesicht-, Lippenkrebsen,

1 Knochentumor,

10 Milzdrisentumoren.

Dabei ist zu bemerken, daß bei einer nicht geringen Zahl der sogenannten ungenügend bestrahlten Patienten auch eine Besserung im Sinne des Zurückgehens der Geschwülste konstatiert werden konnte. Da sich aber ein Teil dieser Patienten unserer weiteren Beobachtung entzogen hat, können sie in unserer Statistik nicht rerwertet werden.

Fassen wir alle unsere bestrahlten Fälle in einer Tabelle zusammen, so ergibt sich:

\begin{tabular}{|c|c|c|c|c|}
\hline Sitz des Tumors & Gesamtzabl & $\begin{array}{c}\text { Ungenügend } \\
\text { bestrablt }\end{array}$ & $\begin{array}{l}\text { Genüge } \\
\text { Nicht } \\
\text { gebessert }\end{array}$ & $\begin{array}{l}\text { estrablt } \\
\text { Vorläufig } \\
\text { gebessert }\end{array}$ \\
\hline $\begin{array}{l}\text { Mamma } \\
\text { Milz-Drüsen-Mediastinum } \\
\text { Magen-Darm } \\
\text { Kopf-Gesicht-Lippe }\end{array}$ & $\begin{array}{r}93 \\
30 \\
19 \\
15 \\
14 \\
12 \\
9 \\
6 \\
4 \\
16 \\
\end{array}$ & $\begin{array}{r}30 \\
9 \\
8 \\
8 \\
11 \\
4 \\
4 \\
4 \\
3 \\
16 \\
\end{array}$ & $\begin{array}{r}49 \\
11 \\
10 \\
4 \\
2 \\
6 \\
3 \\
2 \\
1 \\
-\end{array}$ & $\begin{array}{r}19 \\
10 \\
1 \\
3 \\
1 \\
2 \\
2 \\
- \\
- \\
-\end{array}$ \\
\hline \multirow[t]{2}{*}{$\begin{array}{l}\text { Prophylaktische Bestrah- } \\
\text { lungen }\end{array}$} & $\begin{array}{r}223 \\
7\end{array}$ & 97 & 88 & 38 \\
\hline & 230 & & & \\
\hline
\end{tabular}

Die Resultate der prophylaktischen Bestrahlungen können wir wegen der Kürze der Beobachtungszeit in diesem Bericht nicht berücksichtigen. Von den 97 ungenügend bestrahlten und den 88 nicht gebesserten Fälen werden 34 Patienten noch weiter behandelt. Auch unter diesen Zeitechrift für Krebsforschung. 16, Bd. 1. Heft. 
sind therapeutische Erfolge zu verzeichnen, so daß es möglich ist, daß die Zahl unserer Gebesserten sich noch weiter vermehren wird.

Andererseits ist nicht zu erwarten, daß alle Gebesserten dauernd so bleiben.. Man kann aber hoffen, daß der Prozentsatz der vorläufig Gebesserten, von 126 genügend Bestrahlten $-38=22 \mathrm{pCt}$, auch nach längerer Beobachtungszeit nicht wesentlich sinken wird. Erst nach gründlicher Kenntnis der Wirkungsart der Strahlen, der Anwendung wirksamerer Strahlen und Kombinationen mit geeigneten chemo-therapeutischen Agentien können die Resultate auch bei einem Material, wie das unserige, besser werden.

Von den zahlreichen chemischen Präparaten (Arsenikalien, Enzytol usw.), die wir neben der Röntgenbehandlung den Patienten teils intravenös oder intramuskulär, teils intratumoral appliziert haben, und über die an anderer Stelle ausführlich berichtet wird, erwies sich bis jetzt das Atoxyl im Verein mit arseniger Säure als bestes Adjuvans zur günstigen Beeinflussung des Allgemeinbefindens der Carcinomkranken.

Wir verwenden das Arsen-Atoxyl Marke "Silbe" und injizieren stets intravenös. Nebenschädigungen haben wir nie beobachtet. Eine Verstärkung der carcinomzerstörenden Wirkung der Röntgenstrahlen kommt dem Arsen-Atoxyl, ebensowenig wie allen anderen bis jetzt erprobten chemischen Agentien jedenfalls in den meisten Fällen nicht zu.

Zu der Verbesserung der Strahlenwirkung rechnen wir die Möglichkeit, neben der Geschwulst auch gleichzeitig alle diejenigen Stellen des Körpers zu bestrahlen, in denen sich nach der Erfahrung Metastasen einzustellen pflegen. Das sind z. B. bei Brustdrüsenkrebs die Achselhöhlen, die ganze vordere Thoraxwand, insbesondere auch die Lungen, da Carcinome der Pleura ziemlich häufig sekundär nach Brustkrebs aufzutreten pflegen. Das Ganze ist eine Frage der Zahl der Apparate, die einem Institut zur Verfügung stehen. Wenn wir täglich 40-50 Kranke zu bestrahlen haben, so kann man mit zwei Apparaten nur schwer eine Bestrahlungstherapie in jedem Falle von der Ausdehnung treiben, wie wir es. für nötig halten. Es hat sich nur zu oft gezeigt, daß, wenn wir die sichtbaren Tumoren durch die Bestrahlung zum Verschwinden gebracht hatten, an einer anderen Stelle Metastasen auftraten, die dann schwieriger beeinflußt werden konnten. Es ist absolut nötig, nicht nur die schon fühlbaren und sichtbaren Metastasen durch Röntgenstrahlen zu vernichten, sondern auch diejenigen, die noch so klein sind, daß sie sich der Wahrnehmung entziehen.

Um eine größere Anzahl Patienten nach dieser, unserer Auffassung gemäß, richtigen Art behandeln zu können, haben wir neuerdings einen dritten Röntgenapparat angeschafft, und zwar wiederum eine TriplexApparatur der Siemens \& Halske A.-G., die wir mit der sich bei uns be- 
währten Siederöhre (C. F. H. Müller) betreiben. Ausserdem wird noch ein Zusatzapparat am Triplex eingebaut, der uns ermöglichen wird, die gasfreie Glühkathodenröhre (Coolidge-Röntgenröhre der Siemens \& Halske A.-G.) in Anwendung zu bringen ${ }^{1}$ ).

Zum Schluß noch einige Bemerkungen über die bei uns vorgekommenen Nebenwirkungen der Röntgenstrahlen.

Die von den verschiedenen Therapeuten beobachteten Verschlimmerungen im Zustand der Geschwulstkranken als unmittelbare Folge der Bestrahlungen haben wir bei einigen unserer Geschwulstkranken ebenfalls gesehen. Es handelte sich hierbei nicht um verabfolgte Reizdosen, die die Geschwülste ungünstig beeinflussen, sondern um intensive Bestrahlungen, nach denen die Tumoren mehr oder weniger rapide weicher und kleiner wurden, das Allgemeinbefinden der Patienten sich aber derart verschlimmerte, daB von einer weiteren Behandlung abgesehen werden mußte. Es handelt sich dabei bekanntlich um Intoxikationserscheinungen, die durch den raschen Zerfall der Geschwülste verursacht werden. Es läßt sich aber nicht leugnen, $d a ß$ in manchen, wenn auch glücklicherweise nur wenigen Fällen von langsamem Zurückgehen der Tumoren nach intensiver Bestrahlung, plötzlich eine merkliche Verschlimmerung im Befinden der Patienten auftrat.

Eine Schädigung der blutbildenden Organe haben wir bis jetzt nie beobachtet. Es trat in keinem unserer Fäle eine bedrohliche Leukopenie ein. Da, wo vor der Bestrahlung eine neutrophile Leukozytose oder eine Lymphozytose vorhanden war, sank die Zahl der Leukozyten bzw. der Lymphozyten fast stets bis zur Norm.

Eine besondere Radiosensibilität zeigten die eosinophilen Zellen. Diese verschwanden aus dem Blutbilde stets nach einer Bestrahlungsserie (300 Milliampère-Minuten), ja manchmal sogar nach einer einzigen Bestrahlungssitzung.

Die ausführlichen Untersuchungen über das Blutbild bei bestrahlten Geschwulstkranken, die in unserem Institut von Herrn cand. med. R. Gassul ausgeführt wurden, werden in einer besonderen Abhandlung erscheinen.

Hautschädigungen hatten wir, wie bei der bereits angegebenen geringen Oberflächendosis und der Vielfelderbestrahlung zu erwarten war, nur verhältnismäßig wenige zu verzeichnen. Zur Behandlung der Röntgenulcera und der Erytheme wenden wir mit gutem Erfolge das Eucupin in Form einer 2 proz. Salbe nach folgendem Rezept an:

1) Seit November d. J. sind bei uns zwei Coolidge-Röntgenröhren im Betrieb. Die eine Einrichtung stammt von den Veifa-Werken, die andere von Siemens und Halske. 
20 F. Blumentbal, Bericht üb. d. Tätigkeit im Universitätsinstitut f. Krebsforsch.

Eucupin bihydrochlor. $\quad 2,0$

Solve in Aq. dest. 50,0

Adde Ol. olivar. oder Lanolin,

$$
\text { Vaselin } \overline{a a} \text { ad } 100,0 \text {. }
$$

Das Eucupin ist ein Glied in der homologen Reihe der von Morgenroth erforschten Cininalkaloide, zu denen auch das bekannte pneumokokkenabtötende Optochin gehört. In experimentellen Versuchen am Kaninchenauge ist erwiesen worden, daß das Optochin auch eine an $\ddot{s}$ sthesierende Wirkung ausübt. Diese Anästhesie zeichnet sich vor allem dadurch aus, daß sie, im Vergleich mit dem gebräuchlichsten Anästhetikum. dem Kokain, länger andauert, und daß schwache Lösungen genügen, um eine recht intensive Wirkung zu erzeugen.

Die Untersuchungen der höheren Homologen des Optochins (Aethylhydrokuprein) bezüglich ihrer anästhesierenden Eigenschaften ergaben, daß dem Eucupin (Isoamylhydrokuprein) die höchste anästhesierende Wirkung unter den Chinaalkaloiden zukommt, und daß eine Daueranästhesie durch sehr geringe Konzentrationen dieses Präparats erzielt werden kann.

Wir hielten es demnach für angebracht, das Eucupin bei den häufig mit sehr heftigen Schmerzen einhergehenden Ulzerationen der Haut Krebskranker, die durch Perforation der Geschwulst verursacht werden. anzuwenden $\mathbf{l}$ ).

Die schmerzlindernde Wirkung stellte sich in der Tat in vielen Fïllen sehr sehnell ein. Dabei konnten wir auch eine Reinigung der häufig sehr übelriechenden Geschwürsflächen und eine rasche Leberhäutung der ulzerierten Stellen wahrnehmen. Diese letztere Wirkung verdanken wir zweifellos der bakteriziden Eigenschaft des Eucupins. Es wurde nämlich durch Reagenzglasversuche nachgewiesen ${ }^{2}$ ), daß sowohl Streptokokken wie Staphylokokken durch Eucupin in Verdünnungen von etwa 1:40000 abgetötet werden. Diese Desinfektionswirkung wird durch Gegenwart ron tierischem Gewebe oder gelösten Eiweißsubstanzen nicht beeinträchtigt.

Diese beiden Eigenschaften des Eukupins, die anästhesierende wie die bakterizide, leisten uns auch bei den Röntgenschädigungen der Haut sehr gute Dienste. Obwohl, wie bereits erwähnt, durch Anwendung sehr harter Strahlen und der Vielfelderbestrahlung die Hautschädigungen keine bösartige Form annehmen, so ist es von großem Vorteil, ein Nittel zu haben, durch das wir sowohl die Röntgenulzera wie die Erytheme in vielen Fällen rascher zur Heilung bringen als durch Behandlung mit indifferenten Salben, und dadurch eine Verzögerung in der Röntgenbestrahlung unserer Geschwulstkranken vielfach vermeiden können.

1) J. Tugendreich, Berl. klin. Wochenschr. 1916. No. 10.

2) Morgenroth u. Tugendreich, Berl. klin. Wochenschr. 1916. No. 29. 